\title{
Higher Groups in Homotopy Type Theory
}

\author{
Ulrik Buchholtz \\ Technische Universität Darmstadt \\ Darmstadt, Germany \\ buchholtz@mathematik. \\ tu-darmstadt.de
}

\author{
Floris van Doorn \\ Carnegie Mellon University \\ Pittsburgh, PA, USA \\ fpv@andrew.cmu.edu
}

\author{
Egbert Rijke \\ Carnegie Mellon University \\ Pittsburgh, PA, USA \\ erijke@andrew.cmu.edu
}

\begin{abstract}
We present a development of the theory of higher groups, including infinity groups and connective spectra, in homotopy type theory. An infinity group is simply the loops in a pointed, connected type, where the group structure comes from the structure inherent in the identity types of Martin-Löf type theory. We investigate ordinary groups from this viewpoint, as well as higher dimensional groups and groups that can be delooped more than once. A major result is the stabilization theorem, which states that if an $n$-type can be delooped $n+2$ times, then it is an infinite loop type. Most of the results have been formalized in the Lean proof assistant.
\end{abstract}

\section{CCS CONCEPTS}

- Theory of computation $\rightarrow$ Type theory; Logic and verification; Constructive mathematics;

\section{KEYWORDS}

Higher groups, Homotopy type theory, Lean proof assistant

\section{ACM Reference Format:}

Ulrik Buchholtz, Floris van Doorn, and Egbert Rijke. 2018. Higher Groups in Homotopy Type Theory. In LICS '18: LICS '18: 33rd Annual ACM/IEEE Symposium on Logic in Computer Science, Fuly 9-12, 2018, Oxford, United Kingdom. ACM, New York, NY, USA, 10 pages. https://doi.org/10.1145/ 3209108.3209150

\section{INTRODUCTION}

The homotopy hypothesis is the statement that homotopy $n$-types (topological spaces with trivial homotopy groups above level $n$ ) correspond to $n$-groupoids for $n \in \mathbb{N} \cup\{\infty\}$ via the fundamental $\infty$-groupoid construction. In Grothendieck's original version in Pursuing Stacks [13] this was a conjecture about a particular model of $\infty$-groupoids. It is also a theorem for many particular models of $\infty$-groupoids, for example the Kan simplicial sets, but it is now mostly taken to be a property defining $\infty$-groupoids up to equivalence.

In this paper, we investigate the homotopy hypothesis in the context of homotopy type theory (HoTT). HoTT refers to the homotopical interpretation of Martin-Löf's dependent type theory

Permission to make digital or hard copies of all or part of this work for personal or classroom use is granted without fee provided that copies are not made or distributed for profit or commercial advantage and that copies bear this notice and the full citation on the first page. Copyrights for components of this work owned by others than the author(s) must be honored. Abstracting with credit is permitted. To copy otherwise, or republish, to post on servers or to redistribute to lists, requires prior specific permission and/or a fee. Request permissions from permissions@acm.org.

LICS '18, July 9-12, 2018, Oxford, United Kingdom

(C) 2018 Copyright held by the owner/author(s). Publication rights licensed to ACM ACM ISBN 978-1-4503-5583-4/18/07 ..\$15.00

https://doi.org/10.1145/3209108.3209150
$[2,27]$. In this homotopical interpretation, every type-theoretical construction corresponds to a homotopy-invariant construction on spaces.

In HoTT, every type has a path space given by the identity type. For a pointed type we can construct the loop space, which has the structure of an $\infty$-group. Moreover, if the type is truncated, then we can retreive the usual notion of groups, 2-groups and higher groups. This allows us to define a higher group internally in the language of type theory as a type that is the loop space of a pointed connected type, its delooping.

We also investigate groups that can be delooped more than once, which gives $n$-groups with additional coherences. The full family of groups we consider is in Table 1, which we will explain in detail in section 3. We prove that the first column of this table is correct with respect to the usual formalizations of groups and abelian groups as set-level structures, and we prove the stabilization theorem justifying the ditto marks in the table. Along the way we develop some elementary theory of higher groups and actions thereof.

Our approach is additionally validated by the corresponding observation in $\infty$-topos theory, where it is a theorem that the $\infty$ category of pointed, connected objects in $\mathcal{X}$ is equivalent to the $\infty$-category of higher group objects in $\mathcal{X}$, for any $\infty$-topos $\mathcal{X}[18$, Lemma 7.2.2.11(1)].

We have formalized most of our results in the HoTT library [9] of the Lean Theorem Prover [19]. The formalized results can be found in the file https://github.com/cmu-phil/Spectral/blob/master/ higher_groups.hlean. We will indicate the major formalized results in this paper by referring to the name in the formalization inside square brackets. For more information about the formalization, see section 8.

We are indebted to Michael Shulman for writing a blog post [23] on classifying spaces from a univalent perspective.

\section{PRELIMINARIES}

In this paper we will work in the type theory of the HoTT book [26], although all arguments will also hold in a cubical type theory, such as $[1,7]$. In this section we briefly introduce the concepts we need for the rest of the paper.

The type theory contains dependent function types $(x: A) \rightarrow$ $B(x)$, which are more traditionally denoted as $\Pi_{x: A} B(x)$ and dependent pair types $(x: A) \times B(x)$, which are traditionally denoted as $\Sigma_{x: A} B(x)$. We choose to use this Agda-inspired notation because we often deal with deeply nested dependent sum types.

Within a type $A$ we have the identity type or path type $=_{A}$ : $A \rightarrow A \rightarrow$ Type. We have various operations on paths, such as concatenation $p \cdot q$ and inversion $p^{-1}$ of paths. The functorial action 
Table 1: Periodic table of $k$-tuply groupal $n$-groupoids.

\begin{tabular}{clllll}
\hline$k \backslash n$ & 0 & 1 & 2 & $\cdots$ & $\infty$ \\
\hline 0 & pointed set & pointed groupoid & pointed 2-groupoid & $\cdots$ & pointed $\infty$-groupoid \\
1 & group & 2-group & 3-group & $\cdots$ & $\infty$-group \\
2 & abelian group & braided 2-group & braided 3-group & $\cdots$ & braided $\infty$-group \\
3 & $-»-$ & symmetric 2-group & sylleptic 3-group & $\cdots$ & sylleptic $\infty$-group \\
4 & $-"-$ & $-»-$ & symmetric 3-group & $\cdots$ & $? ? \infty$-group \\
$\vdots$ & $\vdots$ & $\vdots$ & $\vdots$ & $\ddots$ & $\vdots$ \\
$\omega$ & $-”-$ & $-»-$ & $-\cdots-$ & $\cdots$ & connective spectrum \\
\hline
\end{tabular}

of a function $f: A \rightarrow B$ on a path $p: a_{1}={ }_{A} a_{2}$ is denoted $\operatorname{ap}_{f}(p): f\left(a_{1}\right)=f\left(a_{2}\right)$. The constant path is denoted $1_{a}: a=a$.

When a type $A$ is $n$-truncated we write istrunc ${ }_{n} A$. Recall that this is defined by recursion on $n: \mathbb{N}_{-2}:=\mathbb{Z}_{\geq-2}$ :

$$
\begin{aligned}
\text { istrunc }_{-2} A & :=\operatorname{iscontr} A:=(a: A) \times((x: A) \rightarrow(a=x)) \\
\text { istrunc }_{n+1} A & :=(x y: A) \rightarrow \text { istrunc }_{n}(x=y)
\end{aligned}
$$

For any type $A$ we write $\|A\|_{n}$ for its $n$-truncation, i.e., $\|A\|_{n}$ is an $n$-truncated type equipped with a map $|-|_{n}: A \rightarrow\|A\|_{n}$ such that for any $n$-truncated type $B$ the precomposition map

$$
\left(\|A\|_{n} \rightarrow B\right) \rightarrow(A \rightarrow B)
$$

is an equivalence. Then we define being $n$-connected as isconn $n$ $A:=$ iscontr $\|A\|_{n}$. Properties of truncations and connected maps are established in Chapter 7 of [26].

The type of pointed types is Type $\mathrm{pt}_{\mathrm{pt}}:=(A:$ Type $) \times(\mathrm{pt}: A)$. The type of $n$-truncated types is Type ${ }^{\leq n}:=(A$ : Type $) \times$ istrunc $_{n} A$ and for $n$-connected types it is Type ${ }^{>n}:=(A:$ Type $) \times$ isconn $_{n} A$. We will combine these notations as needed.

Given $A$ : Type $_{\mathrm{pt}}$ we define the loop space $\Omega A:=\left(\mathrm{pt}={ }_{A} \mathrm{pt}\right)$, which is pointed with basepoint $1_{\mathrm{pt}}$. The homotopy groups of $A$ are defined to be $\pi_{k} A:=\left\|\Omega^{k} A\right\|_{0}$. These are groups in the usual sense when $k \geq 1$, with neutral element $|1|$ and group operation induced by path concatenation.

Given $A, B$ : Type $_{\mathrm{pt}}$ the type of pointed maps from $A$ to $B$ is $(A \rightarrow \mathrm{pt} B):=(f: A \rightarrow B) \times\left(f(\mathrm{pt})={ }_{B} \mathrm{pt}\right)$. Given $f: A \rightarrow \mathrm{pt} B$ we write $f: A \rightarrow B$ for the first projection (i.e. the underlying map) and $f_{0}: f(\mathrm{pt})=\mathrm{pt}$ for the second projection. The fiber of a pointed map is defined by $\operatorname{fib}(f):=(a: A) \times\left(f(a)={ }_{B} \mathrm{pt}\right)$, which is pointed with basepoint (pt, $f_{0}$ ).

In HoTT we can use higher inductive types to construct EilenbergMacLane spaces $K(G, n)$ [17]. For a group $G$ we define $K(G, 1)$ as the following HIT.

HIT $K(G, 1):=$

• $\star K(G, 1)$;

- $p: G \rightarrow \star=\star$;

- $q:(g h: G) \rightarrow p(g h)=p(g) \cdot p(h)$

- $\epsilon$ : istrunc ${ }_{1} K(G, 1)$.

(Using the univalent universe Type, other direct definitions are also possible, for instance, $K(G, 1)$ is equivalent to the type of small $G$-torsors.) Let $\Sigma X$ denote the suspension of $X$, i.e., the homotopy pushout of $1 \leftarrow X \rightarrow 1$. For an abelian group $A$ can now inductively define $K(A, n+1):=\|\Sigma K(A, n)\|_{n+1}$. Then we have the following result [17].

THeOREM 2.1. Let $G$ be a group and $n \geq 1$, and assume that $G$ is abelian when $n>1$. The space $K(G, n)$ is $(n-1)$-connected and $n$-truncated and there is a group isomorphism $\pi_{n} K(G, n) \simeq G$.

In some of our informal arguments we use the descent theorem for pushouts, ${ }^{1}$ which states that for a commuting cube of types

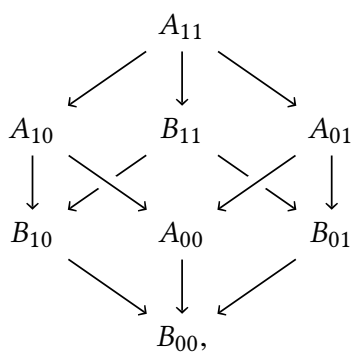

if the bottom square is a pushout and the vertical squares are pullbacks, then the top square is also a pushout. We will use the following slight generalization.

THeorem 2.2. Consider a commuting cube of types as in (1), and suppose the vertical squares are pullback squares. Then the square

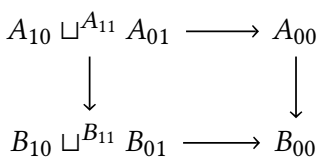

is a pullback square.

Proof. It suffices to show that the pullback

$$
\left(B_{10} \sqcup^{B_{11}} B_{01}\right) \times_{B_{00}} A_{00}
$$

has the universal property of the pushout. This follows by the descent theorem, since by the pasting lemma for pullbacks we also

\footnotetext{
${ }^{1}$ Recall from [18, §6.1.3], following ideas from Charles Rezk, that we can define the $\infty$-toposes among locally cartesian closed $\infty$-categories as those whose colimits are van Kampen, viz., satisfying descent.
} 
have that the vertical squares in the cube

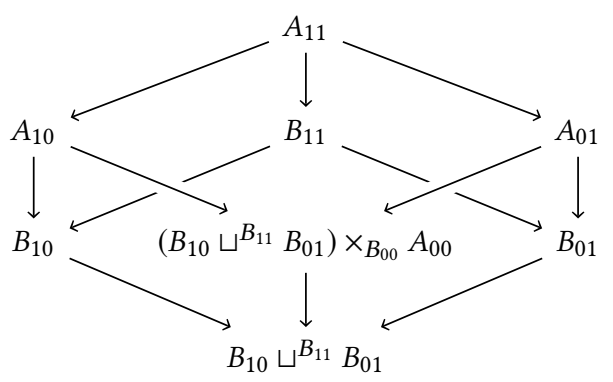

are pullback squares.

In the formalization, arguments using descent are more conveniently done via the equivalent principle captured formally as the flattening lemma [26, §6.12].

\section{HIGHER GROUPS}

Recall that types in HoTT may be viewed as $\infty$-groupoids: elements are objects, paths are morphisms, higher paths are higher morphisms, etc.

It follows that pointed connected types $B$ may be viewed as higher groups, with carrier $\Omega B:=\left(\mathrm{pt}={ }_{B} \mathrm{pt}\right)$. The neutral element is the identity path, the group operation is given by path composition, and higher paths witness the unit and associativity laws. Of course, these higher paths are themselves subject to further laws, etc., but the beauty of the type-theoretic definition is that we don't have to worry about that: all the (higher) laws follow from the rules of the identity types. Writing $G$ for the carrier $\Omega B$, it is common to write $B G$ for the pointed connected type that comes equipped with an identification $G=\Omega B G$. We call $B G$ the delooping of $G$, and we emphasize that it is $B G$ that is the essential datum specifying a higher group.

Let us write

$$
\begin{aligned}
\infty \text {-Group }: & =(G: \text { Type }) \times\left(B G: \text { Type }_{\mathrm{pt}}^{>0}\right) \times(G \simeq \Omega B G) \\
& \simeq\left(G: \text { Type }_{\mathrm{pt}}\right) \times\left(B G: \text { Type }_{\mathrm{pt}}^{>0}\right) \times(G \simeq \mathrm{pt} \Omega B G) \\
& \simeq \text { Type }_{\mathrm{pt}}^{>0}
\end{aligned}
$$

for the type of higher groups, or $\infty$-groups. Note that for $G$ : $\infty$-Group we also have $G$ : Type using the first projection as a coercion. Using the last definition, this is the loop space map, and not the usual coercion!

It is of course common practice in mathematics to refer to a group by the name of its carrier type (a set in ordinary group theory), and it is a familiar fact that the same set may harbor many inequivalent group structures. Likewise, a type may have several different deloopings.

What is perhaps surprising is that even once we fix the H-space structure on a type $A$ there can still be none or infinitely many deloopings of $A$ giving rise to the $\mathrm{H}$-space structure. For example, in classical homotopy theory there are uncountably many loop space structures on the 3-sphere $\mathbb{S}^{3}$ matching its usual $\mathrm{H}$-space structure [20].

We recover the ordinary set-level groups by requiring that $G$ is a 0 -type, or equivalently, that $B G$ is a 1-type. This leads us to introduce

$$
\begin{aligned}
n \text {-Group }: & =\left(G: \text { Type }_{\mathrm{pt}}^{<n}\right) \times\left(B G: \text { Type }_{\mathrm{pt}}^{>0}\right) \times\left(G \simeq_{\mathrm{pt}} \Omega B G\right) \\
& \simeq \text { Type }_{\mathrm{pt}}^{>0, \leq n}
\end{aligned}
$$

for the type of groupal (group-like) (n-1)-groupoids, also known as $n$-groups. For $G$ : 1 -Group a set-level group, we have $B G=K(G, 1)$.

For example, the integers $\mathbb{Z}$ as an additive group are from this perspective represented by their delooping $\mathrm{B} \mathbb{Z}=\mathbb{S}^{1}$, i.e., the circle.

Of course, double loop spaces are even better behaved than mere loop spaces (e.g., they are commutative up to homotopy by the Eckmann-Hilton argument [26, Theorem 2.1.6]). Say a type $G$ is $k$-tuply groupal if we have a $k$-fold delooping, $B^{k} G$ : Type $\mathrm{pt}^{\geq k}$, such that $G=\Omega^{k} B^{k} G$.

Mixing the two directions, let us introduce the type

$$
\begin{aligned}
(n, k) \text { GType } & :=\left(G: \text { Type }_{\mathrm{pt}}^{\leq n}\right) \times\left(B^{k} G: \text { Type }_{\mathrm{pt}}^{\geq k}\right) \times\left(G \simeq_{\mathrm{pt}} \Omega^{k} B^{k} G\right) \\
& \simeq \text { Type } \mathrm{pt}_{\mathrm{pt}}^{\geq k, \leq n+k} \quad \text { [GType_equiv] }
\end{aligned}
$$

for the type of $k$-tuply groupal $n$-groupoids. ${ }^{2}$ (We allow taking $n=$ $\infty$ in which case the truncation requirement is simply dropped. [InfGType_equiv]) Note that $n$-Group $=(n-1,1)$ GType. This shift in indexing is slightly annoying, but we keep it to stay consistent with the literature.

Since there are forgetful maps

$$
(n, k+1) \text { GType } \rightarrow(n, k) \text { GType }
$$

given by $B^{k+1} G \mapsto \Omega B^{k+1} G$ we can also allow $k$ to be infinite, $k=\omega$ by setting

$$
\begin{aligned}
(n, \omega) \text { GType }:= & \lim _{k}(n, k) \text { GType } \\
\simeq & \left(B^{-} G:(k: \mathbb{N}) \rightarrow \text { Type }_{\mathrm{pt}}^{\geq k, \leq n+k}\right) \\
& \quad \times\left((k: \mathbb{N}) \rightarrow B^{k} G \simeq_{\mathrm{pt}} \Omega B^{k+1} G\right) .
\end{aligned}
$$

In section 6 we prove the stabilization theorem (Theorem 6.7), from which it follows that $(n, \omega)$ GType $=(n, k)$ GType for $k \geq n+2$.

When $(n, k)=(\infty, \omega)$, this is the type of stably groupal $\infty$-groups, also known as connective spectra. If we also relax the connectivity requirement, we get the type of all spectra, and we can think of a spectrum as a kind of $\infty$-groupoid with $k$-morphisms for all $k \in \mathbb{Z}$.

The class of higher groups is summarized in Table 1 . We shall prove the correctness of the $n=0$ column in section 5 .

\section{ELEMENTARY OBSERVATIONS}

\subsection{Automorphism groups}

Automorphism groups form a major class of examples of $\infty$-groups. Given any type $A$ and any object $a: A$, the automorphism group at $a$ is defined as automorphism group Aut $a:=(a=a)$. This is indeed an $\infty$-group, because it is the loop space of the connected component of $A$ at $a$, i.e., we define BAut $a:=\operatorname{im}(a: 1 \rightarrow A)=(x: A) \times\|a=x\|_{-1}$. From this definition it is immediate that Aut $a=\Omega$ BAut $a$, so we see that Aut $a$ is indeed an example of an $\infty$-group. Furthermore, if $A$ is $(n+1)$-truncated, then Aut $a$ is an $(n+1)$-group because Aut $a$ is $n$-truncated.

\footnotetext{
${ }^{2}$ This is called $n$ Type $_{k}$ in [3], but here we give equal billing to $n$ and $k$, and we add the " $\mathrm{G}$ " to indicate group-structure.
} 
Moving across the homotopy hypothesis, for every pointed type $(X, x)$ we have the fundamental $\infty$-group of $X, \Pi_{\infty}(X, x):=$ Aut $x$. Its (n-1)-truncation (an instance of decategorification, see section 6) is the fundamental $n$-group of $X, \Pi_{n}(X, x)$, with corresponding delooping $\mathrm{B}_{n}(X, x)=\|$ BAut $x \|_{n}$.

If we take $A=$ Set, we get the usual symmetric groups $S_{n}:=$ $\operatorname{Aut}($ Fin $n)$, where Fin $n$ is a set with $n$ elements. (Note that $B S_{n}=$ $\operatorname{BAut}(\operatorname{Fin} n)$ is the type of all $n$-element sets.) We give further constructions related to ordinary groups in section 7 .

\subsection{Homomorphisms and conjugation}

A homomorphism between higher groups is any function that can be suitably delooped. For $G, H:(n, k)$ GType, we define

$$
\begin{aligned}
\operatorname{hom}_{(n, k)}(G, H):= & (h: G \rightarrow \mathrm{pt} H) \times\left(B^{k} h: B^{k} G \rightarrow \mathrm{pt} B^{k} H\right) \\
& \times\left(\Omega^{k}\left(B^{k} h\right) \sim \mathrm{pt} h\right) \\
\simeq & \left(B^{k} h: B^{k} G \rightarrow \mathrm{pt} B^{k} H\right) .
\end{aligned}
$$

For (connective) spectra we need pointed maps between all the deloopings and pointed homotopies showing they cohere.

Note that if $h, k: G \rightarrow H$ are homomorphisms between set-level groups, then $h$ and $k$ are conjugate if $B h, B k: B G \rightarrow{ }_{\mathrm{pt}} B H$ are freely homotopic (i.e., equal as maps $B G \rightarrow B H$ ).

Also observe that $\pi_{j}\left(B^{k} G \rightarrow \mathrm{pt} B^{k} H\right) \simeq\left\|B^{k} G \rightarrow{ }_{\mathrm{pt}} \Omega^{j} B^{k} H\right\|_{0} \simeq$ $\left\|\Sigma^{j} B^{k} G \rightarrow{ }_{\mathrm{pt}} B^{k} H\right\|_{0}=0$ for $j+k-1 \geq n+k$, that is, for $j>n$, so this suggests that $\operatorname{hom}_{(n, k)}(G, H)$ is $n$-truncated. (The calculation verifies this for the identity component.) To prove this, we need to use an induction using the definition of $n$-truncated. If $f: \operatorname{hom}_{(n, k)}(G, H)$, then its self-identity type is equivalent to $\left(\alpha:\left(z: B^{k} G\right) \rightarrow(f z=f z)\right) \times\left(\alpha \mathrm{pt} \cdot g_{\mathrm{pt}}=f_{\mathrm{pt}}\right)$. This type is no longer a type of pointed maps, but rather a type of pointed sections of a fibration of pointed types.

Definition 4.1. If $X:$ Type $_{\mathrm{pt}}$ and $Y: X \rightarrow$ Type $_{\mathrm{pt}}$, then we introduce the type of pointed sections,

$$
(x: X) \rightarrow p_{\mathrm{pt}} Y x:=(s:(x: X) \rightarrow Y x) \times(s \mathrm{pt}=\mathrm{pt}) .
$$

This type is itself pointed by the trivial section $\lambda x$, pt.

THEOREM 4.2. Let $X:$ Type $_{\mathrm{pt}}^{\geq k}$ be an $(k-1)$-connected, pointed type for some $k \geq 0$, and let $Y: X \rightarrow$ Type $_{\mathrm{pt}}^{\leq n+k}$ be a fibration of $(n+k)$-truncated, pointed types for some $n \geq-1$. Then the type of pointed sections, $(x: X) \rightarrow \mathrm{pt} Y x$, is n-truncated.

[is_trunc_ppi_of_is_conn]

Proof. The proof is by induction on $n$.

For the base case $n=-1$ we have to show that the type of pointed sections is a mere proposition. Since it is pointed, it must in fact be contractible. The center of contraction is the trivial section $s_{0}$. If $s$ is another section, then we get a pointed homotopy from $s$ to $s_{0}$ from the elimination principle for pointed, connected types [26, Lemma 7.5.7], since the types $s x=s_{0} x$ are $(k-2)$-truncated.

To show the result for $n+1$, taking the $n$ case as the induction hypothesis, it suffices to show for any pointed section $s$ that its self-identity type is $n$-truncated. But this type is equivalent to ( $x$ : $X) \rightarrow \mathrm{pt} \Omega(Y x, s x)$, which is again a type of pointed sections, and here we can apply the induction hypothesis.
Corollary 4.3. Let $k \geq 0$ and $n \geq-1$. If $X$ is $(k-1)$-connected, and $Y$ is $(n+k)$-truncated, then the type of pointed maps $X \rightarrow \mathrm{pt} Y$ is $n$-truncated. In particular, $\operatorname{hom}_{(n, k)}(G, H)$ is an n-type for $G, H$ : $(n, k)$ GType.

Corollary 4.4. The type $(n, k)$ GType is $(n+1)$-truncated. [is_trunc_GType]

Proof. This follows immediately from the preceding corollary, as the type of equivalences $G \simeq H$ is a subtype of the homomorphisms from $G$ to $H$.

If $k \geq n+2$ (so we're in the stable range), then $\operatorname{hom}_{(n, k)}(G, H)$ becomes a stably groupal $n$-groupoid. This generalizes the fact that the homomorphisms between abelian groups form an abelian group.

The automorphism group Aut $G$ of a higher group $G:(n, k)$ GType is in $(n, 1)$ GType. This is equivalently the automorphism group of the pointed type $B^{k} G$. But we can also forget the basepoint and consider the automorphism group $\operatorname{Aut}^{c} G$ of $B^{k} G:$ Type ${ }^{\geq k, \leq n+k}$. This now allows for (higher) conjugations. We define the generalized center of $G$ to be $Z G:=\Omega^{k}$ Aut $^{c} G:(n, k+1)$ GType (generalizing the center of a set-level group, see below in subsection 4.4).

\subsection{Group actions}

In this section we consider a fixed group $G$ : GType with delooping $B G$. An action of $G$ on some object of type $A$ is simply a function $X: B G \rightarrow A$. The object of the action is $X(\mathrm{pt}): A$, and it can be convenient to consider evaluation at $\mathrm{pt}: B G$ to be a coercion from actions of type $A$ to $A$. To equip $a: A$ with a $G$-action is to give an action $X: B G \rightarrow A$ with $X(\mathrm{pt})=a$. The trivial action is the constant function at $a$. Clearly, an action of $G$ on $a: A$ is the same as a homomorphism $G \rightarrow$ Aut $a$.

In the case where we take $A:=$ Type, we arrive at the notion of an action $X$ of $G$ on a type: it is a function $X: B G \rightarrow$ Type. In other words, a $G$-type is a type family over $B G$. Then a map of $G$-types from $X$ to $Y$ is just a function $\alpha:(z: B G) \rightarrow X(z) \rightarrow Y(z)$.

If $X$ is a $G$-type, then we can form the

invariants $X^{h G}:=(z: B G) \rightarrow X(z)$, also known as the homotopy fixed points, and the

coinvariants $X_{h G}:=(z: B G) \times X(z)$, which is also known as homotopy orbit space or the homotopy quotient $X / / G$.

It is easy to see that these constructions are respectively the right and left adjoints of the functor that sends a type $X$ to the trivial $G$ action on $X, X^{\text {triv }}: B G \rightarrow$ Type, which is just the constant family at $X$. Indeed, the adjunctions are just the usual argument swap and (un)currying equivalences, for $Y$ : Type,

$$
\begin{aligned}
\operatorname{hom}\left(Y, X^{h G}\right) & =X \rightarrow(z: B G) \rightarrow Y(z) \simeq(z: B G) \rightarrow X \rightarrow Y(z) \\
& \simeq \operatorname{hom}\left(X^{\text {triv }}, Y\right), \\
\operatorname{hom}\left(X_{h G}, Y\right) & =((z: B G) \times X(z)) \rightarrow Y \simeq(z: B G) \rightarrow X(z) \rightarrow Y \\
& \simeq \operatorname{hom}\left(X, Y^{\text {triv }}\right) .
\end{aligned}
$$

If we think of an action $X: B G \rightarrow$ Type as a type-valued diagram on $B G$, this means that the homotopy fixed points and the homotopy orbit space form the homotopy limit and homotopy colimit of this diagram, respectively. 
Proposition 4.5. Let $f: H \rightarrow G$ be a homomorphism of higher groups with delooping $B f: B H \rightarrow \mathrm{pt} B G$, and let $\alpha: \operatorname{hom}(X, Y)$ be a map of G-types. By composing with $f$ we can also view $X$ and $Y$ as $H$-types, in which case we get a homotopy pullback square:

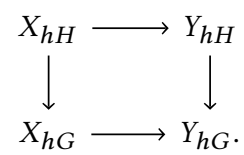

Proof. The vertical maps are induced by $B f$, and the horizontal maps are induced by $\alpha$. The homotopy pullback corner type $C$ is calculated as

$$
\begin{aligned}
C \simeq(z: B G) \times(x: X z) \times(w: B H) \times(y: Y(B f w)) \\
\quad \times(z=B f w) \times(y=\alpha z x) \\
\simeq(w: B H) \times(x: X(B f w))=X_{h H},
\end{aligned}
$$

and under this equivalence the top and the left maps are the canonical ones.

Every group $G$ carries two canonical actions on itself:

the right action $G: B G \rightarrow$ Type, $G(x)=(\mathrm{pt}=x)$, and the the adjoint action $G^{\text {ad }}: B G \rightarrow$ Type, $G^{\text {ad }}(x)=(x=x)$ (by conjugation).

We have $1 / / G=B G, G / / G=1$ and $G^{\text {ad }} / / G=L B G:=\left(\mathbb{S}^{1} \rightarrow B G\right)$, the free loop space of $B G$. Recalling that $\mathrm{B} \mathbb{Z}=\mathbb{S}^{1}$, we see that $G^{\mathrm{ad}}=(\mathrm{BZ} \rightarrow B G)$, i.e., the conjugacy classes of homomorphisms from $\mathbb{Z}$ to $G$. Since the integers are the free (higher) group on one generator, this is just the conjugacy classes of elements of $G$. But that is exactly what we should get for the homotopy orbits of $G$ under the conjugation action.

The above proposition has an interesting corollary:

Corollary 4.6. If $f: H \rightarrow G$ is a homomorphism of higher groups, then $G / / H$ is equivalent to the homotopy fiber of the delooping $B f: B H \rightarrow{ }_{\mathrm{pt}} B G$, where $H$ acts on $G$ via the $f$-induced right action.

Proof. We apply Proposition 4.5 with $\alpha: G \rightarrow 1$ being the canonical map from the right action of $G$ to the action of $G$ on the unit type. Then the square becomes:

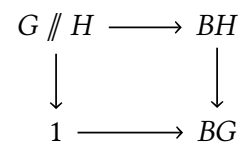

By definition, $B G$ classifies principal $G$-bundles: pullbacks of the right action of $G$. That is, a principal $G$-bundle over a type $A$ is a family $F: A \rightarrow$ Type represented by a map $\chi: A \rightarrow B G$ such that $F(x) \simeq(\mathrm{pt}=\chi(x))$ for all $x: A$.

For example, for every higher group $G$ we have the corresponding Hopf fibration $\Sigma G \rightarrow$ Type represented by the map $\chi_{H}: \Sigma G \rightarrow$ $B G$ corresponding under the loop-suspension adjunction to the identity map on $G$. (This particular fibration can be defined using only the induced H-space structure on $G$.)

This perspective underlies the construction of the first and the third named author of the real projective spaces in homotopy type theory [5]. The fiber sequences $\mathbb{S}^{0} \rightarrow \mathbb{S}^{n} \rightarrow \mathbb{R} \mathrm{P}^{n}$ are principal bundles for the 2-elements group $\mathbb{S}^{0}=S_{2}$ with delooping $B S_{2} \simeq$ $\mathbb{R} P^{\infty}$, the type of 2-element types.

\subsection{Back to the center}

We mentioned the generalized center above and claimed that it generalized the usual notion of the center of a group. Indeed, if $G$ : 1-Group is a set-level group, then an element of $Z G$ corresponds to an element of $\Omega^{2} \mathrm{BAut}^{c} \mathrm{G}$, or equivalently, a map from the 2sphere $\mathbb{S}^{2}$ to Type sending the basepoint to $B G$. By the universal property of $\mathbb{S}^{2}$ as a HIT, this again corresponds to a homotopy from the identity on $B G$ to itself, $c:(z: B G) \rightarrow z=z$. This is precisely a homotopy fixed point of the adjoint action of $G$ on itself, i.e., a central element.

\subsection{Equivariant homotopy theory}

In this section, we would like to clear up a potential source of confusion about the relationship between the classical subject of equivariant homotopy theory of spaces with an action of a topological group $G$ and the homotopy type theory of types with the action of the corresponding object $G$ : GType in the model consisting of homotopy types (for instance presented as simplicial sets); note that $G$ might not be definable in HoTT.

Consider the type $B G \rightarrow$ Type of (small) types with a G-action. Naively, one might think that this represents $G$-equivariant homotopy types, i.e., sufficiently nice ${ }^{3}$ topological spaces with a $G$-action considered up to $G$-equivariant homotopy equivalence. But this is not so.

By Elmendorf's theorem [12], this homotopy theory is rather that of presheaves of (ordinary) homotopy types on the orbit category $O_{G}$ of $G$. This is the full subcategory of the category of $G$-spaces spanned by the homogeneous spaces $G / H$, where $H$ ranges over the closed subgroups of $G$.

Inside the orbit category we find a copy of the group $G$, namely as the endomorphisms of the object $G / 1$ corresponding to the trivial subgroup 1 . Hence, a $G$-equivariant homotopy type gives rise to type with a $G$-action by restriction along the inclusion $B G \hookrightarrow O_{G}$. (Here we consider $B G$ as a (pointed and connected) topological groupoid on one object.)

As remarked by Shulman [24], when $G$ is a compact Lie group, then $O_{G}$ is an inverse EI $\infty$-category, and hence we know how to model type theory in the presheaf $\infty$-topos over $O_{G}$. And in certain simple cases we can even define this model internally. For instance, if $G=\mathbb{Z} / p \mathbb{Z}$ is a cyclic group of prime order, then a small $G$ equivariant type consists of a type with a $G$-action, $X: B G \rightarrow$ Type together with another type family $X^{G}: X^{h G} \rightarrow$ Type, where $X^{G}$ gives for each homotopy fixed point a type of proofs or "special reasons" why that point should be considered fixed [24, 7.6]. Hence the total space of $X^{G}$ is the type of actual fixed points, and the projection to $X^{h G}$ implements the map from actual fixed points to homotopy fixed points.

Even without going to the orbit category, we can say something about topological groups through their classifying types in type theory. For example [6], if $f: H \rightarrow G$ is injective, then the homotopy fiber of $B f$ is by Corollary 4.6 is the homotopy orbit space $G / / H$, which in this case is just the coset space $G / H$, and hence in

\footnotetext{
${ }^{3}$ Sufficiently nice means the $G$-CW-spaces. The same homotopy category arises by taking all spaces with a $G$-action, but then the weak equivalences are the $G$-maps $f: X \rightarrow Y$ that induce weak equivalences on $H$-fixed point spaces $f^{H}: X^{H} \rightarrow Y^{H}$ for all closed subgroups $H$ of $G$.
} 
type theory represents the homotopy type of this coset space. And if

$$
1 \rightarrow K \rightarrow G \rightarrow H \rightarrow 1
$$

is a short exact sequence of topological groups, then $B K \rightarrow B G \rightarrow$ $B H$ is a fibration sequence, i.e., we can recover the delooping $B K$ of $K$ as the homotopy fiber of the map $B G \rightarrow B H$.

\subsection{Some elementary constructions}

If we are given a homomorphism $\varphi: H \rightarrow \operatorname{Aut}(N)$, represented by a pointed map $B \varphi: B H \rightarrow \rightarrow_{\mathrm{pt}} \mathrm{BAut}_{\mathrm{pt}}(B N)$ where $\mathrm{BAut}_{\mathrm{pt}}(B N)$ is the type of pointed types merely equivalent to $B N$, we can build a new group, the semidirect product, $G:=H \ltimes_{\varphi} N$ with classifying type $B G:=(z: B H) \times(B \varphi z)$. The type $B G$ is indeed pointed (by the pair of the basepoint pt in $B G$ and the basepoint in the pointed type $B \varphi(\mathrm{pt}))$, and connected, and hence presents a higher group $G$. An element of $g$ is given by a pair of an element $h: H$ and an identification $g \cdot \mathrm{pt}=\mathrm{pt}$ in $B \varphi(\mathrm{pt}) \simeq_{\mathrm{pt}} B N$. But since the action is via pointed maps, the second component is equivalently an identification $\mathrm{pt}=\mathrm{pt}$ in $B N$, i.e., an element of $N$. Under this equivalence, the product of $(h, n)$ and $\left(h^{\prime}, n^{\prime}\right)$ is indeed $\left(h \cdot h^{\prime}, n\right.$. $\left.\varphi(h)\left(n^{\prime}\right)\right)$.

As a special case we obtain the direct product when $\varphi$ is the trivial action. Here, $B(H \times N) \simeq B H \times B N$.

As another special case we obtain the wreath products $N$ ? $S_{n}$ of a group $N$ and a symmetric group $S_{n}$. Here, $S_{n}$ acts on the direct power $N^{\mathrm{Fin} n}$ by permuting the factors. Indeed, using the representation of $B S_{n}$ as the type of $n$-element types, the map $B \varphi$ is simply $A \mapsto(A \rightarrow B N)$. Hence the delooping of the wreath product $G:=N \imath S_{n}$ is just $B G:=\left(A: B S_{n}\right) \times(A \rightarrow B N)$.

\section{SET-LEVEL GROUPS}

In this section we give a proof that the $n=0$ column of Table 1 is correct. Note that for $n=0$ the hom-types $\operatorname{hom}_{(0, k)}(G, H)$ are sets, which means that $(0, k)$ GType forms a 1-category. Let Group be the category of ordinary set-level groups (a set with multiplication, inverse and unit satisfying the group laws) and AbGroup the category of abelian groups.

THEOREM 5.1. We have the following equivalences of categories (for $k \geq 2)$ :

$$
\begin{aligned}
& (0,1) \text { GType } \simeq \text { Group; } \quad \text { [cGType_equivalence_Grp] } \\
& (0, k) \text { GType } \simeq \text { AbGroup. [cGType_equivalence_AbGrp] }
\end{aligned}
$$

Since this theorem has been formalized we will not give all details of the proof.

Proof. Let $k \geq 1$ and $G$ be a group which is abelian if $k>1$ and let $X:$ Type $_{\mathrm{pt}}^{\geq k, \leq k}$. If we have a group homomorphism $\varphi$ : $G \rightarrow \Omega^{k} X$ we get a map $e_{\varphi}^{k}: K(G, k) \rightarrow$ pt $X$. For $k=1$ this follows directly from the induction principle of $K(G, 1)$. For $k>1$ we can define the group homomorphism $\widetilde{\varphi}$ as the composite $G \stackrel{\varphi}{\rightarrow}$ $\Omega^{k} X \simeq \Omega^{k-1}(\Omega X)$, and apply the induction hypothesis to get a map $e_{\widetilde{\varphi}}^{k-1}: K(G, k-1) \rightarrow{ }_{\mathrm{pt}} \Omega X$. By the adjunction $\Sigma \dashv \Omega$ we get a pointed map $\Sigma K(G, k-1) \rightarrow{ }_{\text {pt }} X$, and by the elimination principle of the truncation we get a map $K(G, k)=\|\Sigma K(G, k-1)\|_{k} \rightarrow$ pt $X$.
We can now show that $\Omega^{k} e_{\varphi}^{k}$ is the expected map, that is, the following diagram commutes, but we omit this proof here.

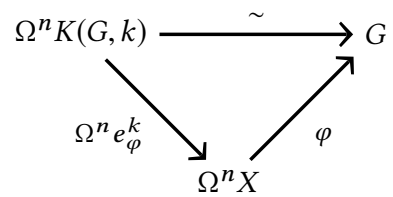

Now if $\varphi$ is a group isomorphism, by Whitehead's Theorem for truncated types [26, Theorem 8.8.3] we know that $e_{\varphi}^{k}$ is an equivalence, since it induces an equivalence on all homotopy groups (trivially on the levels other than $k$ ). We can also show that $e_{\varphi}^{k}$ is natural in $\varphi$.

Note that if we have a group homomorphism $\psi: G \rightarrow G^{\prime}$, we also get a group homomorphism $G \rightarrow \Omega^{k} K\left(G^{\prime}, k\right)$, and by the above construction we get a pointed map $K(\psi, k): K(G, k) \rightarrow{ }_{\text {pt }} K\left(G^{\prime}, k\right)$. This is functorial, which follows from naturality of $e_{\varphi}^{k}$.

Finally, we can construct the equivalence explicitly. We have a functor $\pi_{k}:(0, k)$ GType $\rightarrow$ AbGroup which sends $G$ to $\pi_{k} B G$. Conversely, we have the functor $K(-, k):$ AbGroup $\rightarrow(0, k)$ GType. We have natural isomorphisms $\pi_{k} K(G, k) \simeq G$ by Theorem 2.1 and $K\left(\pi_{k} X, k\right) \simeq_{\mathrm{pt}} X$ by the application of Whitehead described above. The construction is exactly the same for $k=1$ after replacing AbGroup by Group.

\section{STABILIZATION}

In this section we discuss some constructions with higher groups [3]. We will give the actions on the carriers and the deloopings, but we omit the third component, the pointed equivalence, for readability. We recommend keeping Table 1 in mind during these constructions.

decategorification Decat : $(n, k)$ GType $\rightarrow(n-1, k)$ GType $\left\langle G, B^{k} G\right\rangle \mapsto\left\langle\|G\|_{n-1},\left\|B^{k} G\right\|_{n+k-1}\right\rangle$

discrete categorification Disc $:(n, k)$ GType $\rightarrow(n+1, k)$ GType $\left\langle G, B^{k} G\right\rangle \mapsto\left\langle G, B^{k} G\right\rangle$

These functors make $(n, k)$ GType a reflective sub- $(\infty, 1)$-category of $(n+1, k)$ GType. That is, there is an adjunction Decat $\dashv$ Disc [Decat_adjoint_Disc] ${ }^{4}$ such that the counit induces an isomorphism DecatoDisc $=$ id [Decat_Disc]. These properties are straightforward consequences of the universal property of truncation.

There are also iterated versions of these functors.

$\infty$-decategorification $\infty$-Decat $:(\infty, k)$ GType $\rightarrow(n, k)$ GType $\left\langle G, B^{k} G\right\rangle \mapsto\left\langle\|G\|_{n},\left\|B^{k} G\right\|_{n+k}\right\rangle$

discrete $\infty$-categorification $\infty$-Disc $:(n, k)$ GType $\rightarrow(\infty, k)$ GType $\left\langle G, B^{k} G\right\rangle \mapsto\left\langle G, B^{k} G\right\rangle$

These functors satisfy the same properties: $\infty$-Decat $\dashv \infty$-Disc [InfDecat_adjoint_InfDisc] such that the counit induces an isomorphism $\infty$-Decat $\circ \infty$-Disc $=$ id [InfDecat_InfDisc] .

For the next constructions, we need the following properties.

Definition 6.1. For $A:$ Type $_{\mathrm{pt}}$ we define the $n$-connected cover of $A$ to be $A\langle n\rangle:=\operatorname{fib}\left(A \rightarrow\|A\|_{n}\right)$. We have the projection $p_{1}$ : $A\langle n\rangle \rightarrow \mathrm{pt} A$.

${ }^{4}$ In the formalization the naturality of the adjunction is a separate statement, [Decat_adjoint_Disc_natural]. This is also true for the other adjunctions. 
LEMMA 6.2. The universal property of the $n$-connected cover states the following. For any $n$-connected pointed type $B$, the pointed map

$$
(B \rightarrow \mathrm{pt} A\langle n\rangle) \rightarrow{ }_{\mathrm{pt}}(B \rightarrow \mathrm{pt} A),
$$

given by postcomposition with $p_{1}$, is an equivalence.

[connect_intro_pequiv]

Proof. Given a map $f: B \rightarrow{ }_{\mathrm{pt}} A$, we can form a map $\tilde{f}: B \rightarrow$ $A\langle n\rangle$. First note that for $b: B$ the type $|f b|_{n}={ }_{\|A\|_{n}}|\mathrm{pt}|_{n}$ is $(n-1)$ truncated and inhabited for $b=$ pt. Since $B$ is $n$-connected, the universal property for connected types shows that we can construct a $q b:|f b|_{n}=|\mathrm{pt}|_{n}$ for all $b$ such that $q_{0}: q b_{0} \cdot \mathrm{ap}_{|-|_{n}}\left(f_{0}\right)=1$. Then we can define the map $\widetilde{f}(b):=(f b, q b)$. Now $\widetilde{f}$ is pointed, because $\left(f_{0}, q_{0}\right):\left(f b_{0}, q b_{0}\right)=\left(a_{0}, 1\right)$

Now we show that this is indeed an inverse to the given map. On the one hand, we need to show that if $f: B \rightarrow \mathrm{pt} A$, then $p_{1} \circ \widetilde{f}=f$. The underlying functions are equal because they both send $b$ to $f(b)$. They respect points in the same way, because $\operatorname{ap}_{p_{1}}\left(\widetilde{f}_{0}\right)=f_{0}$. The proof that the other composite is the identity follows from a computation using fibers and connectivity, which we omit here, but can be found in the formalization.

The next reflective sub- $(\infty, 1)$-category is formed by looping and delooping.

looping $\Omega:(n, k)$ GType $\rightarrow(n-1, k+1)$ GType

$\left\langle G, B^{k} G\right\rangle \mapsto\left\langle\Omega G, B^{k} G\langle k\rangle\right\rangle$

delooping $\mathrm{B}:(n, k)$ GType $\rightarrow(n+1, k-1)$ GType

$\left\langle G, B^{k} G\right\rangle \mapsto\left\langle\Omega^{k-1} B^{k} G, B^{k} G\right\rangle$

We have $\mathrm{B} \dashv \Omega$ [Deloop_adjoint_Loop], which follows from Lemma 6.2 and $\Omega \circ \mathrm{B}=$ id [Loop_Deloop], which follows from the fact that $A\langle n\rangle=A$ if $A$ is $n$-connected.

The last adjoint pair of functors is given by stabilization and forgetting. This does not form a reflective sub- $(\infty, 1)$-category.

forgetting $F:(n, k)$ GType $\rightarrow(n, k-1)$ GType $\left\langle G, B^{k} G\right\rangle \mapsto\left\langle G, \Omega B^{k} G\right\rangle$

stabilization $S:(n, k)$ GType $\rightarrow(n, k+1)$ GType $\left\langle G, B^{k} G\right\rangle \mapsto\left\langle S G,\left\|\Sigma B^{k} G\right\|_{n+k+1}\right\rangle$, where $S G=\left\|\Omega^{k+1} \Sigma B^{k} G\right\|_{n}$

We have the adjunction $S \dashv F$ [Stabilize_adjoint_Forget] which follows from the suspension-loop adjunction $\Sigma \dashv \Omega$ on pointed types.

The next main goal in this section is the stabilization theorem, stating that the ditto marks in Table 1 are justified.

The following corollary is almost [26, Lemma 8.6.2], but proving this in Book HoTT is a bit tricky. See the formalization for details.

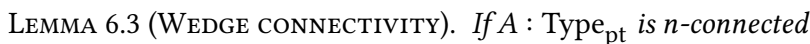
and $B:$ Type $_{\mathrm{pt}}$ is m-connected, then the map $A \vee B \rightarrow A \times B$ is $(n+m)$-connected. [is_conn_fun_prod_of_wedge]

Let us mention that there is an alternative way to prove the wedge connectivity lemma: Recall that if $A$ is $n$-connected and $B$ is $m$-connected, then $A * B$ is $(n+m+2)$-connected [21, Theorem 6.8]. Hence the wedge connectivity lemma is also a direct consequence of the following lemma.

Lemma 6.4. Let $A$ and $B$ be pointed types. The fiber of the wedge inclusion $A \vee B \rightarrow A \times B$ is equivalent to $\Omega A * \Omega B$.
Proof. Note that the fiber of $A \rightarrow A \times B$ is $\Omega B$, the fiber of $B \rightarrow A \times B$ is $\Omega A$, and of course the fiber of $1 \rightarrow A \times B$ is $\Omega A \times \Omega B$. We get a commuting cube

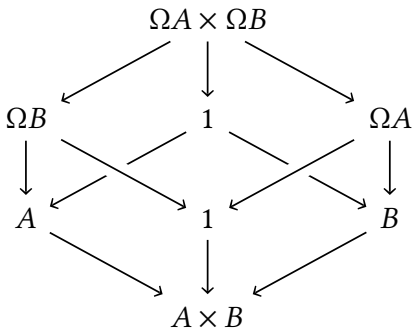

in which the vertical squares are pullback squares.

By the descent theorem for pushouts it now follows that $\Omega A * \Omega B$ is the fiber of the wedge inclusion.

The second main tool we need for the stabilization theorem is:

Theorem 6.5 (Freudenthal). If $A:$ Type $_{\mathrm{pt}}^{>n}$ with $n \geq 0$, then the map $A \rightarrow \Omega \Sigma A$ is $2 n$-connected.

This is [26, Theorem 8.6.4].

The final building block we need is:

Lemma 6.6. There is a pullback square

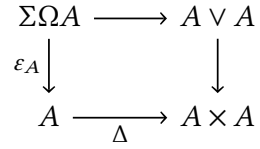

for any $A$ : Type $_{\mathrm{pt}}$.

Proof. Note that the pullback of $\Delta: A \rightarrow A \times A$ along either inclusion $A \rightarrow A \times A$ is contractible. So we have a cube

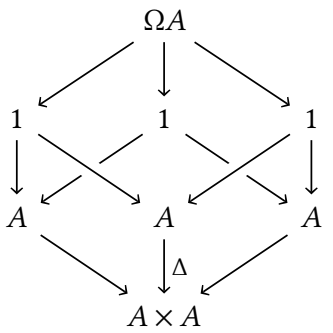

in which the vertical squares are all pullback squares. Therefore, if we pull back along the wedge inclusion, we obtain by the descent theorem for pushouts that the square in the statement is indeed a pullback square.

Theorem 6.7 (Stabilization). If $k \geq n+2$, then $S:(n, k)$ GType $\rightarrow$ $(n, k+1)$ GType is an equivalence, and any $G:(n, k)$ GType is an infinite loop space. [stabilization]

Proof. We show that $F \circ S=$ id $=S \circ F:(n, k)$ GType $\rightarrow$ $(n, k)$ GType whenever $k \geq n+2$.

For the first, the unit map of the adjunction factors as

$$
B^{k} G \rightarrow \Omega \Sigma B^{k} G \rightarrow \Omega\left\|\Sigma B^{k} G\right\|_{n+k+1}
$$

where the first map is $2 k-2$-connected by Freudenthal, and the second map is $n+k$-connected. Since the domain is $n+k$-truncated, the composite is an equivalence whenever $2 k-2 \geq n+k$. 
For the second, the counit map of the adjunction factors as

$$
\left\|\Sigma \Omega B^{k} G\right\|_{n+k} \rightarrow\left\|B^{k} G\right\|_{n+k} \rightarrow B^{k} G
$$

where the second map is an equivalence. By the two lemmas above, the first map is $2 k-2$-connected.

For example, for $G:(0,2)$ GType an abelian group, we have $B^{n} G=K(G, n)$, an Eilenberg-MacLane space.

The adjunction $S \dashv F$ implies that the free group on a pointed set $X$ is $\Omega\|\Sigma X\|_{1}=\pi_{1}(\Sigma X)$. If $X$ has decidable equality, $\Sigma X$ is already 1-truncated. It is an open problem whether this is true in general.

Also, the abelianization of a set-level group $G: 1$-Group is $\pi_{2}(\Sigma B G)$. If $G:(n, k)$ GType is in the stable range $(k \geq n+2)$, then $S F G=G$.

\section{PERSPECTIVES ON ORDINARY GROUP THEORY}

In this section we shall indicate how the theory of higher groups can yield a new perspective even on ordinary group theory.

From the symmetric groups $S_{n}$, we can get other finite groups using the constructions of subsection 4.6. Other groups can be constructed more directly. For example, $B A_{n}$, the classifying type of the alternating group, can be taken to be the type of $n$-element sets $X$ equipped with a sign ordering: this is an equivalence class of an ordering Fin $n \simeq X$ modulo even permutations. Indeed, there are only two possible sign orderings, so this definition corresponds to first considering the short exact sequence

$$
1 \rightarrow A_{n} \rightarrow S_{n} \stackrel{\text { sgn }}{\longrightarrow} S_{2} \rightarrow 1
$$

where the last map is the sign map, then realizing the sign map as given by the map Bsgn : $B S_{n} \rightarrow B S_{2}$ that takes an $n$-element set to its set of sign orderings, and finally letting $B A_{n}$ be the homotopy fiber of Bsgn.

Similarly, $B C_{n}$, the classifying type of the cyclic group on $n$ elements, can be taken to be the type of $n$-elements sets $X$ equipped with a cyclic ordering: an equivalence class of an ordering Fin $n \simeq X$ modulo cyclic permutations. But unlike the above, where we had the coincidence that $\operatorname{Aut}\left(S_{2}\right) \simeq S_{2}$, this doesn't correspond to a short exact sequence. Rather, it corresponds to a sequence

$$
1 \rightarrow C_{n} \rightarrow S_{n} \rightarrow \operatorname{Aut}(\operatorname{Fin}(n-1)) \simeq S_{(n-1) !}
$$

where the delooping of the last map is the map from $B S_{n}$ to $B S_{(n-1) !}$ that maps an $n$-element set to the set of cyclic orderings, of which there are $(n-1)$ ! many - since once we fix the position in the ordering of a particular element, we are free to permute the rest.

As another example, consider the map $p: B S_{4} \rightarrow \mathrm{pt} B S_{3}$ that maps a 4-element set $X$ to its set of 2-by-2 partitions, of which there 3. Using this construction, we can realize some famous semidirect and wreath product identities, such as $A_{4} \simeq S_{2}^{2} \rtimes A_{3}, S_{4} \simeq S_{2}^{2} \rtimes S_{3}$, and, for the octahedral group, $O_{h} \simeq S_{2}^{3} \rtimes S_{3} \simeq S_{2}$ 々 $S_{3}$.

Let us turn to a different way of getting new groups from old, namely via covering space theory.

\subsection{1-groups and covering spaces}

The connection between covering spaces of a pointed connected type $X$ and sets with an action of the fundamental group of $X$ has already been established in homotopy type theory [15]. Let us recall this connection and expand a bit upon it.

For us, a pointed connected type $X$ is equivalently an $\infty$-group $G: \infty$-Group with delooping $B G:=X$. A covering space over $B G$ is simply a type family $C: B G \rightarrow$ Set that lands in the universe of sets. Hence by our discussion of actions in subsection 4.3 it is precisely a set with a $G$-action. Since Set is a 1-type, $C$ extends uniquely to a type family $C^{\prime}:\|B G\|_{1} \rightarrow$ Set, but $\|B G\|_{1}$ is the delooping of the fundamental group of $X$, and hence $C^{\prime}$ is the uniquely determined choice of a set with an action of the fundamental group.

The universal covering space is the simply connected cover of $B G$,

$$
\widetilde{B G}: B G \rightarrow \text { Set, } \quad z \mapsto\|\mathrm{pt}=z\|_{0} .
$$

Note that the total space of $\widetilde{B G}$ is indeed the 1-connected cover $B G\langle 1\rangle$, since $\left\|\mathrm{pt}={ }_{B G} \mathrm{pt}\right\|_{0} \simeq\left(|\mathrm{pt}|=_{\|B G\|_{1}}|\mathrm{pt}|\right)$. Also note that if $G$ is already a 1-group, then this is just the right action of $G$ on itself, and in general, it is the right action of $G$ on the fundamental group (i.e., the decategorification of $G$ ) via the truncation homomorphism from $G$ to $\pi_{1}(B G)$, where we can also view $\pi_{1}(B G)$ as the 1-Group decategorification of $G$.

In general, there is a Galois correspondence between connected covers of $B G$ and conjugacy classes of subgroups of the fundamental group. Indeed, if $C: B G \rightarrow$ Set has a connected total space, then the space $\left(g:\|B G\|_{1}\right) \times C^{\prime}(g)$ is itself a connected, 1-truncated type, and the projection to $\|B G\|_{1}$ induced an inclusion of fundamental groups once a point $\mathrm{pt}: C^{\prime}(\mathrm{pt})$ has been chosen.

Theorem 7.1 (Fundamental THEOREM OF GALOIS THEORY For COVERING SPACES).

(1) The automorphism group of the universal covering space $\widetilde{B G}$ is isomorphic to the 1-group decategorification of $G$,

$$
\operatorname{Aut}(\widetilde{B G}) \simeq \operatorname{Decat}_{1}(G) \simeq \pi_{1}(B G) .
$$

(2) Furthermore, there is a contravariant correspondence between conjugacy classes of subgroups of $\operatorname{Decat}_{1}(G)$ and connected covers of $B G$.

(3) This lifts to a Galois correspondence between subgroups of Decat $_{1}(G)$ and pointed, connected covers of BG. The normal subgroups correspond to Galois covers.

Note that the universal covering space and the trivial covering space (constant at the unit type) are canonically pointed, reflecting the fact that the two trivial subgroups are normal.

The first part of the fundamental theorem has a clear generalization to higher groups:

THEOREM 7.2 (FundAMENTAL THEOREM OF GALOIS THEORY For $n$-COVERS, PART ONE). The automorphism group of the universal n-type cover $U_{n}(B G)$,

$$
U_{n}(B G): B G \rightarrow \text { Type }^{\leq n}, \quad z \mapsto\|\mathrm{pt}=z\|_{n}
$$

of $B G$ is isomorphic to the $(n+1)$-group decategorification of $G$,

$$
\operatorname{Aut}\left(U_{n}(B G)\right) \simeq \operatorname{Decat}_{n+1}(G) \simeq \Pi_{n+1}(B G) .
$$

Proof. Note that BAut $\left(U_{n}(B G)\right)$ is the image of the map $1 \rightarrow$ $\left(B G \rightarrow\right.$ Type $\left.^{\leq n}\right)$ that sends the canonical element to $U_{n}(B G)$. Since $B G$ is connected, this image is exactly $\|B G\|_{n+1}$ by [21, Theorem 7.1]. Then we are done, since $\mathrm{B} \Pi_{n+1}(B G) \simeq\|B G\|_{n+1}$, by definition. 
It is possible to use the other parts of Theorem 7.1 in order to define the notions of "subgroup" and normal "subgroup" for $n$ groups, which then become structure on rather than a property of a homomorphism $f: K \rightarrow G$. Since any map is an inclusion up to homotopy, we refrain from using the word "subgroup". Instead, we say that a normal structure on $f$ is a delooping $B(G / / K)$ of the type $G / / K$ together with a map $B q: B G \rightarrow$ pt $B(G / / K)$ giving rise to a fiber sequence

$$
G / / K \rightarrow B K \stackrel{B f}{\longrightarrow} B G \stackrel{B q}{\longrightarrow} B(G / / K) .
$$

\subsection{Central extensions and group cohomology}

The cohomology of a higher group $G$ is simply the cohomology of its delooping $B G$. Indeed, for any spectrum $A$, we define

$$
H_{\mathrm{Grp}}^{k}(G, A):=\left\|B G \rightarrow \rightarrow_{\mathrm{pt}} B^{k} A\right\|_{0} .
$$

Of course, to define the $k^{\prime}$ th cohomology group, we only need the $k$-fold delooping $B^{k} A$.

If $A:(\infty, 2)$ GType is a braided $\infty$-group, then we have the second cohomology group $H_{\mathrm{Grp}}^{2}(G, A)$, and an element $c: B G \rightarrow \mathrm{pt} B^{2} A$ gives rise to a central extension

$$
B A \rightarrow B H \rightarrow B G \stackrel{c}{\rightarrow} B^{2} A,
$$

where $B H$ is the homotopy fiber of $c$. This lifts to the world of higher groups the usual result that isomorphism classes of central extensions of a 1-group $G$ by an abelian 1-group $A$ are given by cohomology classes in $H_{\text {Grp }}^{2}(G, A)$.

In the Spectral repository there is full formalization of the Serre spectral sequence for cohomology [8]. If we have any normal subgroup fiber sequence for $\infty$-groups as in (2), then we get a corresponding spectral sequence with $E_{2}$-page

$$
H_{\mathrm{Grp}}^{p}\left(G / / K, H_{\mathrm{Grp}}^{q}(K, A)\right)
$$

and converging to $H_{\mathrm{Grp}}^{n}(G, A)$, where $A$ is any truncated, connective spectrum, which could even be a left $G$-module, in which case we reproduce the Hochschild-Serre spectral sequence.

\section{FORMALIZATION}

We have formalized many results of this paper. We use the proof assistant Lean $2^{5}$. This is an older version of the proof assistant Lean $^{6}$ (version 3.3 as of January 2018). We use the old version, since the newer version doesn't officially support HoTT, although there is an experimental library for $\mathrm{HoTT}^{7}$, but that doesn't have as much theory as the library in Lean 2.

The Lean 2 HoTT library is divided into two parts, the core library $^{8}$ and the formalization of spectral sequences ${ }^{9}$. We worked in the latter, so that we could use the results from that repository, such as theorems about Eilenberg-MacLane spaces and pointed maps. All results in this paper are stated in one file ${ }^{10}$, although for many results the main parts of the proof is elsewhere (in Emacs, click on a name and press $\mathrm{M}-$. to find a definition).

\footnotetext{
${ }^{5}$ https://github.com/leanprover/lean2

${ }^{6} \mathrm{https}: / /$ leanprover.github.io/

${ }^{7}$ https://github.com/gebner/hott3

${ }^{8}$ https://github.com/leanprover/lean2/blob/master/hott/hott.md

${ }^{9}$ https://github.com/cmu-phil/Spectral

${ }^{10}$ https://github.com/cmu-phil/Spectral/blob/master/higher_groups.hlean
}

To build the file, install Lean 2 via the instructions from that repository, and then download the Spectral repository and compile it (you can use the command path/to/lean2/bin/linja on the command-line to compile the library you're in). The Spectral repository contains some unproven results, marked by sorry. These are however not related to the results discussed in this paper, and you can write print axioms theoremname in a file to verify that sorry isn't used in the proofs.

\section{CONCLUSION}

We have presented a theory and formalization of higher groups in HoTT, and we have proved that for set-level structures we recover the well-known objects: groups and abelian groups. A possible next step would be to do the same for the 1-type objects. The corresponding algebraic objects have a long history. Strict 2-groups predate category theory as they originate in Whitehead's study of crossed modules [28]. The theory of weak 2-groups was begun by Grothendieck's student Hoàng Xuân Sính [25] and further developed in [4]. It should be possible to prove within HoTT that weak 2 -groups and crossed modules are equivalent to 2-groups in our sense, when we use the respective, correct notions of equivalence.

Symmetric 2-groups are by the stabilization theorem the same as 1-truncated symmetric spectra. These are described more simply than arbitrary crossed modules as Picard groupoids. This is part of the stable homotopy hypothesis $[14,16]$. It should also be possible to develop the theory of Picard groupoids in HoTT, and thus prove the corresponding stable homotopy hypothesis.

Higher groups have been intensively studied in homotopy theory, in particular after $p$-completion for $p$ a prime. A $p$-compact group is an $\mathbb{F}_{p}$-local $\infty$-group whose carrier is $\mathbb{F}_{p}$-finite, see [10]. They are good homotopical analogues of Lie groups, and they interact nicely with compact Lie groups, for instance:

Theorem 9.1 ([11]). Let $P$ be a p-toral group, and let $G$ be a compact Lie group. Then $\left\|B P \rightarrow{ }_{\mathrm{pt}} B G\right\|_{0}$ is isomorphic to the conjugacy classes of homomorphisms from $P$ to $G$.

Higher groups also play a particularly prominent role in the development of quantum field theory in cohesive homotopy type theory [22]. In cohesive type theory we can actually capture the topological or smooth structure of groups and their classifying types, and hence develop Lie theory properly, including the higher group generalization thereof. All of our results only use the core part of HoTT, and hence they remain valid also in cohesive HoTT.

Note that we have crucially used a trick to study higher groups in HoTT, namely that these can be represented by pointed, connected types. The alternative would have been to define them as group-like algebras for the little $k$-cubes operad $E_{k}$. But this requires exactly the kind of infinitary tower of coherence conditions that we don't yet know how to define in HoTT. (Or whether it is even possible.) Thus, while we have the type of higher groups, we do not have the type of higher monoids (general $E_{k}$-algebras). Thus their theory, and the corresponding stabilization theorem, is currently beyond the reach of HoTT.

\section{ACKNOWLEDGMENTS}

The authors gratefully acknowledge the support of the Air Force Office of Scientific Research through MURI grant FA9550-15-1-0053. 
The authors would also like to thank the Isaac Newton Institute for Mathematical Sciences for its support and hospitality during the program "Big Proof" when part of the work on this paper was done; the program was supported by Engineering and Physical Sciences Research Council under grant number EP/K032208/1. Any opinions, findings and conclusions or recommendations expressed are those of the authors and do not necessarily reflect the views of any sponsoring institution, government or any other entity.

\section{REFERENCES}

[1] Carlo Angiuli, Robert Harper, and Todd Wilson. 2017. Computational Higherdimensional Type Theory. In Proceedings of the 44th ACM SIGPLAN Symposium on Principles of Programming Languages (POPL 2017). ACM, New York, NY, USA, 680-693. https://doi.org/10.1145/3009837.3009861

[2] Steve Awodey and Michael A. Warren. 2009. Homotopy theoretic models of identity types. Math. Proc. Cambridge Philos. Soc. 146, 1 (2009), 45-55. https: //doi.org/10.1017/S0305004108001783 arXiv:0709.0248

[3] John C. Baez and James Dolan. 1998. Categorification. In Higher category theory (Evanston, IL, 1997). Contemp. Math., Vol. 230. Amer. Math. Soc., Providence, RI, 1-36. https://doi.org/10.1090/conm/230/03336

[4] John C. Baez and Aaron D. Lauda. 2004. Higher-dimensional algebra. V: 2-Groups. Theory Appl. Categ. 12 (2004), 423-491. arXiv:math/0307200

[5] Ulrik Buchholtz and Egbert Rijke. 2017. The real projective spaces in homotopy type theory. In 32nd Annual ACM/IEEE Symposium on Logic in Computer Science (LICS 2017). IEEE, New York, NY, USA, 1-8. https://doi.org/10.1109/LICS.2017. 8005146

[6] Omar Antolín Camarena. 2017. The homotopy fiber of the map on classifying spaces. Retrieved Jan. 28, 2018 from http://www.matem.unam.mx/omar/notes/ hofib-grphom.html

[7] Cyril Cohen, Thierry Coquand, Simon Huber, and Anders Mörtberg. 2016. Cubical Type Theory: a constructive interpretation of the univalence axiom. In $21 s t$ International Conference on Types for Proofs and Programs (TYPES 2015). Schloss Dagstuhl. Leibniz-Zent. Inform., Wadern. arXiv:1611.02108 To appear.

[8] Floris van Doorn, Jeremy Avigad, Steve Awodey, Ulrik Buchholtz, Egbert Rijke, and Mike Shulman. 2018. Spectral Sequences in Homotopy Type Theory. (2018). https://github.com/cmu-phil/Spectral Paper forthcoming.

[9] Floris van Doorn, Jakob von Raumer, and Ulrik Buchholtz. 2017. Homotopy Type Theory in Lean. In Interactive Theorem Proving (ITP 2017). Springer, Cham, 479-495. https://doi.org/10.1007/978-3-319-66107-0_30

[10] William G. Dwyer and Clarence W. Wilkerson. 1994. Homotopy fixed-point methods for Lie groups and finite loop spaces. Ann. Math. (2) 139, 2 (1994), 395-442. https://doi.org/10.2307/2946585

[11] William G. Dwyer and Alexander Zabrodsky. 1987. Maps between classifying spaces. In Algebraic topology, Barcelona, 1986. Lecture Notes in Math., Vol. 1298. Springer, Berlin, 106-119. https://doi.org/10.1007/BFb0083003

[12] Anthony D. Elmendorf. 1983. Systems of fixed point sets. Trans. Am. Math. Soc. 277 (1983), 275-284. https://doi.org/10.2307/1999356

[13] Alexander Grothendieck. 1983. Pursuing Stacks. http://thescrivener.github.io/ PursuingStacks/ Manuscript.

[14] Nick Gurski. 2018. The Stable Homotopy Hypothesis and Categorified Abelian Groups. Retrieved Jan. 30, 2018 from https://golem.ph.utexas.edu/category/ 2018/01/the_stable_homotopy_hypothesis.html Blog post. The $n$-Category Café

[15] Kuen-Bang Hou (Favonia) and Robert Harper. n.d.. Covering Spaces in Homotopy Type Theory. In 22nd International Conference on Types for Proofs and Programs (TYPES 2016) (Leibniz International Proceedings in Informatics (LIPIcs)), Herman Geuvers, Silvia Ghilezan, and Jelena Ivetic (Eds.). Schloss Dagstuhl-LeibnizZentrum für Informatik, Dagstuhl, Germany.

[16] Niles Johnson and Angélica M. Osorno. 2012. Modeling stable one-types. Theory and Applications of Categories 26, 20 (2012), 520-537. http://www.tac.mta.ca/tac/ volumes/26/20/26-20abs.html

[17] Daniel R. Licata and Eric Finster. 2014. Eilenberg-MacLane Spaces in Homotopy Type Theory. In Proceedings of the foint Meeting of the Twenty-Third EACSL Annual Conference on Computer Science Logic (CSL) and the Twenty-Ninth Annual ACM/IEEE Symposium on Logic in Computer Science (LICS) (CSL-LICS '14). ACM, New York, NY, USA, Article 66, 9 pages. https://doi.org/10.1145/2603088.2603153

[18] Jacob Lurie. 2009. Higher topos theory. Annals of Mathematics Studies, Vol. 170 Princeton University Press, Princeton, NJ. xviii+925 pages. https://doi.org/10. $1515 / 9781400830558$

[19] Leonardo de Moura, Soonho Kong, Jeremy Avigad, Floris van Doorn, and Jakob von Raumer. 2015. The Lean Theorem Prover (System Description). In Automated Deduction - CADE-25, P. Amy Felty and Aart Middeldorp (Eds.). Springer, Cham, 378-388. https://doi.org/10.1007/978-3-319-21401-6_26

[20] David L. Rector. 1971. Loop structures on the homotopy type of $S^{3}$. (1971), 99-105. Lecture Notes in Math., Vol. 249. https://doi.org/10.1007/BFb0060900
[21] Egbert Rijke. 2017. The join construction. arXiv:1701.07538 Preprint

[22] Urs Schreiber and Michael Shulman. 2014. Quantum Gauge Field Theory in Cohesive Homotopy Type Theory. In Proceedings 9th Workshop on Quantum Physics and Logic, Brussels, Belgium, 10-12 October 2012 (Electronic Proceedings in Theoretical Computer Science), Ross Duncan and Prakash Panangaden (Eds.), Vol. 158. Open Publishing Association, Waterloo, NSW, 109-126. https://doi.org/ 10.4204/EPTCS.158.8

[23] Michael Shulman. 2015. The Univalent Perspective on Classifying Spaces. Retrieved Jan. 29, 2018 from https://golem.ph.utexas.edu/category/2015/01/the_ univalent_perspective_on_c.html Blog post. The $n$-Category Café.

[24] Michael Shulman. 2017. Univalence for inverse EI diagrams. Homology, Homotopy and Applications 19, 2 (2017), 219-249. https://doi.org/10.4310/HHA.2017.v19.n2. a12 arXiv: 1508.02410

[25] Hoàng Xuân Sính. 1975. Gr-catégories. Ph.D. Dissertation. Université Paris Diderot (Paris 7). Advisor(s) Grothendieck, Alexander. http://www.iaz.uni-stuttgart.de/ LstAlg/Kuenzer/Kuenzer/sinh.html

[26] The Univalent Foundations Program. 2013. Homotopy Type Theory: Univalent Foundations of Mathematics. http://homotopytypetheory.org/book/, Institute for Advanced Study. arXiv:1308.0729

[27] Vladimir Voevodsky. 2006. A very short note on homotopy $\lambda$-calculus. (2006). http://www.math.ias.edu/ vladimir/Site3/Univalent_Foundations_files/ Hlambda_short_current.pdf

[28] J. H. C. Whitehead. 1949. Combinatorial homotopy. II. Bull. Am. Math. Soc. 55 (1949), 453-496. https://doi.org/10.1090/S0002-9904-1949-09213-3 